\title{
Doing patient-centredness versus achieving public health targets: $A$ critical review of interactional dilemmas in ART adherence support.
}

\section{Abstract}

Anti-retroviral Therapy (ART) transformed HIV into a chronic disease but its individual and public health benefits depend on high levels of adherence. The large and rising number of people on ART, now also used as prevention, puts considerable strain on health systems and providers in low and middle as well as high-income countries, which are our focus here. Delivering effective adherence support is thus crucial but challenging, especially given the promotion of patient-centredness and shared decision making in HIV care. To illuminate the complexities of ART adherence support delivered in and through clinical encounters, we conducted a multi-disciplinary interpretative literature review. We reviewed and synthesized 82 papers published post 1997 (when ART was introduced) belonging to three bodies of literature: public health and psychological studies of ART communication; anthropological and sociological studies of ART; and conversation analytic studies of patient-centredness and shared decision-making. We propose three inter-related tensions which make patientcentredness particularly complex in this infectious disease context: achieving trust versus probing about adherence; patient-centredness versus reaching public health targets; and empowerment versus responsibilisation as 'therapeutic citizens'. However, there is a dearth of evidence concerning how precisely ART providers implement patient-centredness, shareddecision making in practice, and enact trust and therapeutic citizenship. We show how conversation analysis could lead to new, actionable insights in this respect.

Key-words: Patient-centredness; shared decision-making; therapeutic citizenship; HIV; ART; adherence; adherence support; conversation analysis 


\section{Introduction}

HIV is an infectious disease of major global importance, which was transformed in the late 1990s from a deadly to a chronic disease by the advent of anti-retroviral therapy (ART). Globally, the number of people on ART is now large (19.5 million in 2016), and still rising in low, middle and high income settings (UNAIDS, 2017), the latter of which is our focus here. The rise is partly due to a shift in guidelines: clients should now commence life-long ART when diagnosed and 'ready' for treatment, whatever their CD4 count (WHO, 2016). Moreover, evidence that ART can prevent onward transmission has led to 'treatment as prevention' (TasP) and pre-exposure prohylaxis (PrEP) approaches (Yin et al., 2014). Despite controversy (Kelen \& Cresswell, 2017), PrEP is now offered to those at substantial risk of exposure, including 'key populations' (Men who have Sex with Men, MSM; sex workers), sero-discordant couples (where only one partner is HIV positive), and pregnant women (WHO, 2016).

As a result, ART takes up considerable resources and places an increasing burden on already constrained health systems and providers. One contribution to this pressure is the need for continuous monitoring and support of ART adherence (BHIVA, 2016; WHO, 2016). Suboptimal adherence can lead to drug resistance, thereby threatening the drugs' individual and public health (and economic) benefits. Hence, adherence has been called the 'Achilles heel' of ART (Nachega et al., 2014). A significant minority of clients do not achieve optimal adherence (Mills et al., 2006a; Kirwan et al., 2016). For example, even in a relatively high performing country like the UK, approximately $10 \%$ of HIV infected people have a viral load associated with life-threatening risks to the individual and wider public (Nachega et al., 2014; 
Kirwan et al., 2016). Moreover, vulnerable groups such as people under 25, the less affluent, prisoners and substance misusers, have lower adherence levels (BHIVA, 2016; Kirwan et al., 2014). PrEP has extended concerns about suboptimal adherence to people at risk of contracting HIV (Gupta et al., 2013). Thus, high quality HIV adherence support is a health policy priority, and a recognized challenge given that clients are increasing in number, but also age and diversity (Scottish Government 2009; WHO, 2016).

Adherence support can be delivered in the community or clinic; in this paper, we focus on professional adherence support, delivered in the clinical encounter (de Bruin et al., 2010a, b). The clinical encounter, and more specifically, the provider-client relationship, has been identified as one of the correlates of adherence, alongside other factors relating to the client (e.g. health status; illness beliefs); the treatment regime (complexity, side-effects), and the socio-economic and health system context (poverty, access) (Ammassari et al., 2002; Ickovics \& Meade, 2002; Vervoort, Borleffs, Hoepelman, \& Grypdonck, 2007). We seek to illuminate the role of provider-client relationships and communication as key areas of adherence support, and in particular the roles of patient-centredness and shared decisionmaking. These have been dominant health paradigms in high income settings since the 1990s, are part of global (WHO, 2016) and national (BHIVA, 2016) HIV policies, and have a direct, significant and recognised bearing on interaction (BHIVA, 2016). Patient-centredness places the patient's feelings and preferences at the heart of the consultation and treatment decisions (Stewart, 2001); shared decision-making is similarly characterised by basing decisions on patients' values or preferences, and by providing options and establishing partnerships (Makoul \& Clayman, 2006). These approaches entail a shift away from a paternalistic focus on achieving compliance with providers' instructions to the pursuit of concordance and respect for clients' autonomy and informed choice, even if this entails treatment rejection (Kremer, Bader, O’Cleirigh, Bierhoff \& Brockmeyer, 2004). Reasons for the shift include 
cost-effectiveness; some evidence suggests that shared decision-making enhances treatment adherence and health outcomes (Coulter, 1997; Matthias, Salyers \& Frankel, 2013). However, the evidence is not unequivocal and the shift is also ideological: treating patients and professionals as equals is seen as a moral 'good' (Pilnick \& Dingwall 2011).

However, patient-centredness and shared decision-making merit more critical reflection. For example, Mol (2008) argued that the 'logic of choice' underpinning shared decision-making is an overly rationalistic approach, promulgated by western neo-liberal ideals which celebrate autonomy, but which does not necessarily constitute 'good' care. Others have argued against a 'one size fits all' model; whether and how patient-centredness and shared decision-making principles work in practice should be examined within specific socio-cultural, health system and disease contexts (Matthias, Salyers \& Frankel, 2013; Pilnick \& Zayts, 2015). Pilnick and Dingwall (2011) have critiqued the universal pursuit of patientcentredness, arguing that asymmetry may simply be endemic to the medical enterprise given the professionals' greater medical expertise, and it may ultimately be our view of this asymmetry that is problematic rather than its continued presence.

To date, most studies examining and supporting patient-centredness and shared decision-making have focused on diseases for which several alternative management options exist (e.g. diabetes, cancer), and where patients' views about treatment can be given precedence without the possibility of causing wider harms to public health. The situation is rather different in the context of infectious diseases such as HIV. Here, patients' decisions not to follow treatment advice may have particularly large public health consequences. Therefore, we seek to unpack the complexities of patient-centred care and shared decision-making in adherence support in the HIV context, and address the following questions. What is the relationship between ART adherence and provider-patient communication, in particular patient-centeredness and shared decision-making, in high income countries? How, if at all, are 
patient-centeredness and shared decision-making put into practice in ART adherence support provided in the clinical encounter? We will show how ethnographic studies can aid our understanding of the complexities and dilemmas involved and we propose that conversation analysis (CA) can provide an empirical understanding of how these dilemmas play out in practice.

We base our arguments on a multi-disciplinary systematic literature review guided by principles of critical interpretative synthesis (Dixon-Woods et al., 2006), the details of which we present below.

\section{Methodology}

Critical interpretative synthesis (CIS; Dixon-Woods et al., 2005) is an iterative form of review which seeks to develop theoretical and empirically grounded understandings of a phenomenon through critical interpretation and synthesis of diverse bodies of quantitative and qualitative literature. We combined an aggregative review (summarizing existing knowledge), with an interpretative review (developing new interpretations) (Gough, Thomas, \& Oliver, 2012).

\section{Search strategy}

We reviewed three separate 'sets' of literature, identified through different search and selection strategies informed by emerging insights, in keeping with CIS principles (DixonWoods et al., 2006). We started with set 1: empirical studies of the relationship between ART adherence and provider-client interaction and relationships in high income settings, mostly produced by psychologists and public health experts, and mostly focused on the US. This set $(\mathrm{N}=44)$ was generated by a systematic search, using key words (Table 1$)$ to search databases 
(CINAHL, PubMed, MEDLINE, PsychINFO and Scopus) in 2012, 2014 and 2017. Search terms were adjusted after an initial search and screening of titles and abstracts (e.g. we added 'antiretroviral' to 'ART OR ARV').

\section{INSERT Table 1. Keywords (in abstracts).}

Findings regarding the complexity and sensitivity of adherence talk drew our attention to set 2 , the predominantly ethnographic anthropological and sociological literature which theorizes ART, its social and moral dimensions and its impact on identities and relationships. Studies of therapeutic citizenship (Nguyen, 2004, 2005) were particularly key here. These papers $(\mathrm{N}=10)$ were identified through snowball sampling, starting from Nguyen's (2004) seminal publication. They helped to interpret and conceptualise the empirical findings from set 1 .

Third, our review indicated that the analysis of actual clinical encounters was highly relevant but largely absent in both sets; we therefore turned to conversation analytic (CA) studies. These use recorded interactions to examine patient-centredness and shared decisionmaking. Snowball sampling was again used for this third set $(\mathrm{N}=28)$ to explore the potential contribution of this kind of analysis to our emerging understanding of challenges in adherence support.

\section{In- and exclusion}

We included English language publications after 1997, when highly active ARVs (HAART) were introduced. All study designs, types of health providers (e.g. pharmacists) and clients (e.g. children, prisoners) were eligible for inclusion. We excluded studies from low and middle income countries (LMICs) in set 1 because our interest was in assessing current 
knowledge regarding the role of provider-client relationships and communication in ART adherence in high income settings. However, in set 2, we did include studies from LMICs, as this is where most ethnographic research is conducted, and we used this set to develop theoretical insights and arguments rather than to synthesize empirical findings. Theoretical insights emerging from studies conducted in LMICs are likely transferable to high income countries (HICs), although this requires further assessment through empirical studies. Virtually all CA studies are conducted in HICs, but set 3 also included a few studies from LMICs, which were relevant since our purpose was to demonstrate the kinds of insights the methodology of CA could provide into provider-client interaction, patient-centredness and shared decision-making. CIS acknowledges that methodologically weak papers may still offer conceptual insights and recommends only excluding papers with 'fatal flaws' (Dixon-Woods et al., 2006), but we did not encounter any in our sample.

For set 1, a research assistant screened titles and abstracts of papers for relevance and applied inclusion and exclusion criteria. The first author read the abstracts and full papers of those included and checked a sample of the excluded papers to ensure agreement on exclusion criteria. Papers were excluded usually because they were not written in English, discussed the 'art' of communication but not ART, or addressed 'interaction' between ART correlates, not provider-client interaction.

\section{Sampling}

The search for set 1 resulted in 194 studies. Following CIS principles (Dixon-Woods et al., 2004), we sought to be comprehensive but not exhaustive and used purposive (maximum variation) and theoretical sampling (Gough, Oliver \& Thomas, 2012). For instance, we ensured that we selected studies including less common populations (e.g. conducted in countries other than US; $100 \%$ adherent clients). We applied principles of theoretical 
saturation: the review of the full papers continued until coherent conclusions emerged from the studies reviewed and no substantially new or contradictory insights appeared in other studies. Moreover, although the new studies reviewed in 2017 added nuances, alternative examples and so on, they reinforced our conclusions and therefore our judgement that we had reached a form of saturation and our conclusions were robust. In addition, in set 2, we selected studies which provided theoretical insights and tools, such as 'therapeutic citizenship' which appeared to resonate with, and were able to explain, some of the empirical findings reported across several studies. Figure 1 depicts the search results.

\section{INSERT Figure 1.}

\section{Data extraction and synthesis}

For set 1, we used a data-extraction table (exemplified in Supplemental table 1) to record primary data (statistics and interview quotes) and the original researchers' interpretations. We used these to develop, with the help of sets 2 and 3, new synthesizing interpretations which encompass and go beyond insights from the individual studies (cf. Dixon-Woods et al. 2006). For sets 2 and 3 we produced mainly written summaries (like Dixon-Wood et al. 2006), since ethnographic studies, reflective commentaries (set 2) and detailed conversation analyses (set

3) do not lend themselves for systematic 'data-extraction'. Moreover, we used sets 2 and 3 mainly to develop our own synthesizing interpretations and arguments (presented in sections 3.3. and 3.4), rather than for systematic empirical synthesis.

We first present our synthesis of set 1 , and discuss the evidence regarding the association between provider-client relationships, communication and adherence (Section 3.1). We subsequently focus more specifically on the role of patient-centredness and shared decision-making (section 3.2). We then discuss the complexities and tensions involved in adherence support and use the anthropological and sociological literature from set 2 to further 
articulate the tensions and interpersonal, moral dilemmas in adherence support and communication (3.3). Finally, we discuss how detailed studies of interaction can aid our understanding of the enactment of patient-centeredness and shared decision-making in the area of HIV/ART (section 3.4). We conclude by highlighting the need for methods which allow the examination of actual adherence communication as it happens, rather than post-hoc assessments of it.

\section{Findings}

\section{1 The role of provider-client relationships and communication in ART adherence.}

A number of studies and systematic reviews indicate that provider-client relationships can foster or impede adherence (Alfonso et al., 2006, 2009; Atkinson, Schonnesson, Williams \& Timpson, 2008; Oetzel, Wilcox, Avila Hill, Archipoli \& Ginossar, 2015; Schneider, Kaplan,

Greenfield, Li, \& Wilson, 2004; Van den Berg, Neilands, Mallory, Johnson, Chen, \& Saberi, 2016; Vervoort, Borleffs, Hoepelman \& Grypdonck, 2007, Mills et al., 2006a). The evidence is not unequivocal however. Some of the studies included in the systematic review by Ammassari and colleagues (2002) did not establish a relationship between the provider-client relationship and adherence. In addition, some non-adherent clients report a good relationship with their provider (Kremer et al., 2004).

Communication appears an important dimension of the provider-client relationship affecting adherence and, more generally, client satisfaction. First, studies which asked what aspects of the provider-client relationship enhance patient satisfaction, adherence, or both, identified features such as providers showing respect (Alfonso et al., 2009; Murri et al., 2002; Tugenberg, Ware \& Wyatt, 2006; Vervoort et al., 2006); understanding, openness, and honesty (Alfonso et al., 2009); a caring attitude and responsiveness (Vervoort et al., 2006); 
'reflective listening' (Wilson et al., 2010); and taking time to listen (Vervoort et al., 2006). These aspects all relate to communication. Second, providers' ability to address adherence barriers, whether clients' psychological characteristics (e.g. self-efficacy, 'treatment readiness'), treatment factors (e.g. side effects), or structural issues (e.g. housing, poverty), relies on open discussion of these barriers (cf. Mills et al. 2006a). Third, adherence support requires accurate assessment of (non) adherence, usually based on a combination of CD4 and viral load monitoring, pill counting and self-report (WHO, 2016). However, providers appear to commonly over and under-estimate adherence, especially amongst lower educated and unemployed clients (Murri et al., 2002). Accuracy of providers' adherence assessment will partly depend on the quality of provider-client communication.

Three key features of doctor-patient communication and relationships recur in the literature on ART adherence: trust, patient-centredness, and joint decision making. It is therefore to the review of these features we now turn.

\subsection{Trust, patient-centredness and shared-decision making: Achievable ideals?}

First, trust, which will partly depend on communication features like taking time to listen (Vervoort et al., 2006), is found to be a facilitator of adherence in quantitative studies (Altice, Mostashari, \& Friedland, 2001; Blackstok et al., 2012; Saha et al, 2010) and qualitative and mixed-method reviews (Mills et al., 2006; Vervoort et al., 2006). Altice, Mostashari and Friedland (2001) found that trust in the provider, and to a lesser extent in the medication and medical institution, were correlated with treatment acceptance amongst US prisoners. However, Graham, Shahani, Grimes, Hartman and Giordano (2015) found adherence was not predicted by trust in physicians nor in the healthcare system among newly diagnosed patients, although physician trust was associated with retention in care. 
Levels of trust vary per client group, with members of marginalised groups generally having lower levels of trust in providers, treatment and institutions (Pach et al., 2003; Saha et al., 2010, Siegel et al., 2000). For example, in an interview study with drug users (88\% African American), some respondents expressed suspicions about being experimented on because 'people don't like dope friends' (Pach et al., 2003, p. 92). Distrust may well have contributed to the very high non-adherence rate $(75 \%)$ in this study. Similarly, Gilbert et al. (2007, p. 166) asked participants with a history of drug-abuse to complete the prompt 'When I am deciding about taking my HIV medications, I think about . . . . Several statements reflected compromised trust in the government, medical institutions and provider, such as: 'Lack of trust when the doctor terminates medications that are working' (op cit., p. 170). Black Americans' trust in HIV health care providers has been found to be generally lower than white clients', likely related to prior experiences of discrimination in everyday life and health care (Saha et al., 2010).

Second, turning to consider patient-centredness, several studies have found that patients' perception of being known 'as a person' (often regarded as key to patientcentredness) predicts ART adherence (Schneider et al., 2004; Beach, Keruly \& Moore, 2006). Alfonso et al. (2009, p.122) describe how providers identified a range of patient-centred attitudes and behaviours that enhanced communication and trust, including 'putting themselves in the patients' shoes', addressing specific needs, treating people as individuals, and 'hearing the whole person and not just the symptoms'. Patients also appreciated information being matched to their needs. Hence, patient-centredness may affect adherence through other correlates such as trust, or psychological characteristics like health optimism or self-efficacy (Atkinson et al., 2008).

Third, there is also some evidence that shared decision-making is associated with adherence, but the evidence is more mixed. In a qualitative study of German clients who 
refused treatment (Kremer et al. 2004, p. 66), several clients had felt coerced by their provider to take treatment. They critiqued providers' unilateral decisions and pursuit of compliance (e.g. 'The doctor should not act as a general ordering patients to take pills. Those who do not obey are lost. They have nothing to say'). A Swedish survey study (Nilsson Schönnesson, Diamond, Ross, Williams \& Bratt, 2006) found that perceived pressures from medical staff to take HIV medication correlated with reduced adherence. Mills et al.'s (2006b) systematic review found some evidence (four studies) that active involvement in treatment decision making facilitates adherence, but this was far less strong than for trust (29 studies). Lewis, Colbert, Erlen \& Meyers (2006) found that a common feature of clients with perfect adherence was that they had established an active partnership with their physicians and identified themselves as part of the health care team. Alfonso et al. (2009) found that some clients reported that collaboration and involvement in treatment decisions was empowering and sustained adherence; providers too found this important. However, these two studies included non-representative client groups; primarily white and relatively highly educated. Active involvement may be a general preference of these client groups, rather than a general determinant of adherence, which as Lewis et al. (2006) suggest may have been fostered by other factors such as high education levels or cohabitation (providing social support). Marelich and Murphy's (2003) study also point to the variable importance of shared decisionmaking; clients who were less ill indicated greater desire to be actively involved in medical management. Finally, in a cross-sectional survey with 554 predominantly white clients, Schneider et al. (2004) found that out of seven physician-patient relationship quality variables, only participatory decision-making was not significantly associated with ART adherence.

In summary, evidence suggests that providers and clients commonly deem patientcentredness and shared decision-making important, in general and for adherence, but there is 
variation across patient groups. Moreover, most studies rely on cross-sectional surveys or interviews; few studies analyse actual interactions. Those that do, suggest a tendency for adherence discussions to lack features of patient-centredness and shared-decision making. For example, Barfod et al. (2006) observed and took fieldnotes on 183 consultations in the USA and Denmark (66\% white clients), and interviewed physicians. They found that most consultations included adherence discussions, but they lacked depth. The authors identified the use of what they described as leading questions ('you don't have any problems, do you?') or broad, standard questions rather than individualized ones, often resulting in superficial responses of 'low believability'. Probing was not common, and physicians responded to such statements with 'okaying' or 'circumventing dialogue', described as continuing the discussion without drawing attention to a potential 'lie'. However, the use of field notes in this study means that details of these interactions and the sequential relationship between patient and physician talk are not available. Barton Laws and colleagues (2013a, b) analysed recorded consultations with 435 patients and 45 providers in the US, and found that providers asked mainly what were categorised as closed and leading questions. Providers checked for understanding infrequently, asked few questions which elicited values, opinions or preferences, and used more 'directives' in adherence talk compared to other topics. Clients asked fewer questions in these sections of the consultation. 'Resolution processes', including treatment decisions and problem solving included few expressions of patients' values or needs (Barton Laws et al., 2013b). On the contrary, problem solving often contained directives (Wilson et al., 2010), and joint problem solving was uncommon even when patients reported adherence problems (Barton Laws et al. 2013a). Callon et al. (2016, p. 1112) found that 'a substantial minority of [US] providers ( $24 \%$ ) did not ask or elicit accurate disclosure from patients who were asked'. 
Given the observed limitations in depth, space for clients' questions or expressions of needs, and joint problem solving, these observational studies indicate that patient-centredness and shared decision-making are not routinely prominent in ART adherence discussions. Indeed, clients report that providers prioritise the need to adhere over their choice and concerns (Kremer et al. 2003). Similarly, Stevenson et al. (2000) found little evidence that patients and general practitioners shared information about, or views on, medicines (not specifically ART). Hence, they had no basis on which to share treatment-decisions.

These are important findings. Despite mixed evidence regarding their causal impact on adherence, it is nonetheless clear that clients, providers and policy-makers commonly deem patient-centredness important for good relationships and communication, and for adherence. It also appears important for client satisfaction (Johnston Roberts 2000; Kremer et al., 2004; Okoro \& Odedina, 2016), which is crucial given the need to retain clients with HIV in lifelong care (WHO, 2016).

In the next section we discuss three tensions, or interactional dilemmas, which we suggest may limit the space of shared decision-making and patient-centredness in ART adherence discussions.

\subsection{ART adherence and the clinical encounter: Tensions and morality}

We have seen that in addition to trust, patient-centredness and shared decision-making are widely regarded as important elements for achieving good adherence and satisfactory provider-client support. Yet, evidence regarding their effectiveness and actual implementation are mixed. We propose that this ambiguity can be understood in terms of three related interactional tensions between conflicting demands: probing versus trust; public health targets versus patient-centredness; and responsibilisation versus empowerment. 
The first tension arises from the need to maintain trust and to establish whether clients have been taking prescribed medication, which requires discussion and probing. In one study (Barfod et al., 2006), providers explained that one reason for limited adherence discussion is that they feel awkward about discussing adherence, especially when clients have previously reported good adherence, there are no 'objective' signs of non-adherence, or the relationship appears fragile and providers worry about creating guilt and damaging trust. Discussing adherence with clients belonging to marginalized groups such as drug users, prisoners or ethnic minorities may be particularly complex and risky. As mentioned, they have generally more limited trust in providers (Pach, Cerbone, \& Gerstein, 2003; Saha, Jacobs, Moore, \& Beach, 2010; Siegel, Karus, \& Schrimshaw, 2000) and in the healthcare system (Pellowski, Price, Allen, Eaton \& Kalichman, 2017). Moreover, when discussing the potential impact of 'deviant' behaviours (e.g. substance abuse) on adherence, perceived moral judgements regarding such behaviours may compound those associated with non-adherence (de Kok, Laurier \& Widdicombe, 2012).

The dilemma of whether to trust or at least avoid questioning the client, or whether to probe their adherence, can be seen as part of a second, broader dilemma: the dual directive to achieve patient-centredness and public health targets (Watermeyer \& Penn, 2012). Providers are asked to be patient-centred and involve patients in decisions (BHIVA, 2016; WHO, 2016), seen in some contexts as patients' (legal) right (e.g. in Scotland: the Patient Rights (Scotland) Act 2011). At the same time, professionals are expected to work towards public health targets, making adherence a priority. Several authors have argued that ART has led to a remedicalisation of HIV, with providers' focus shifting from quality of life to treatment success, from personalized (palliative) care to technical monitoring of viral load, and from people to drugs (Rosengarten, Hart, Flowers \& Imrie, 2004; Yallop et al. 2002). Tugenberg, Ware and Wyatt (2006) note providers' sense of responsibility for clients' adherence, fuelled by the 
urgency of a deadly epidemic. This may explain observed provider responses to (suspected) poor adherence such as lecturing, becoming angry or shaming clients (op cit.), and more generally the paternalistic pursuit of compliance (Bader et al, 2006; Kremer et al., 2004).

For clients, however, social, psychological, or practical problems (e.g. poor mental health, homelessness) may trump adherence concerns. Perceived differential priorities and the feeling that non-medical challenges are not legitimate issues to raise in consultations may obstruct discussion of adherence barriers (de Kok, Laurier \& Widdicombe, 2012; Rosengarten et al., 2004). A further barrier to discussion of adherence problems is that clients fear providers' disapproval, anger, or even loss of interest and care if not following 'Doctor's orders' (Tugenberg et al. 2006, p. 271). Reluctance to share adherence difficulties may be enhanced, ironically, by providers' emphasis on adherence and clients' good relationship with their provider, whom they do not want to disappoint (Tugenberg et al., 2006; Yallop, Lowth, Fitzgerald, Reid \& Morelli, 2002). Yet, sharing adherence difficulties and beliefs about medication is central to adherence support (Poppa et al., 2004; Schneider et al., 2004) and shared decision-making.

Various features of the disease and policy context therefore complicate patientcentredness in adherence conversations. Moreover, providers' sense of responsibility for, and emphasis on, clients' adherence may not only be a barrier to patient-centredness and shared decision-making; it may introduce a third tension, between empowerment and responsibilisation.

In line with western, neo-liberal ideals of individual autonomy, patient-centredness and shared decision-making are often assumed to be desirable because they are 'empowering' (cf. Mol, 2008). Models, like the Healthcare Empowerment model, suggest that improved adherence will occur when patients are 'empowered' or engaged, informed, committed, collaborative and tolerant of uncertainty (Van den Berg et al., 2016). However, sociological 
and anthropological studies raise questions about the empowering effects of shared decisionmaking, by illuminating how new forms of treatment like ART, and new treatment paradigms, change identities, social relationships, perceived rights and responsibilities.

Rosengarten et al. (2004) note how ART, like other medical technologies, has come to reveal new 'truths' about our bodies (e.g. CD 4 counts), triggering new obligations to self and others: keeping the viral load down (cf. Novas \& Rose, 2000). Providers and family members may expect that people living with HIV 'work at' staying well, and thus adhere, in return for their care (Ware et al. 2009). Kagee et al. (2014) note public health practitioners' tendency to 'responsibilise' HIV clients, by framing adherence as 'prosocial behaviour' which good citizens ought to perform to reduce the societal and health system burden. This sense of obligation to adhere is captured in Nguyen's (2004) much used notion of 'therapeutic citizenship'; a shared illness identity associated with rights (e.g. treatment access) and responsibilities (e.g. adherence). It appears then that by fulfilling a 'duty' to adhere, people living with HIV can maintain a moral identity (e.g. being a good and responsible client, relative, partner).

Specific forms of therapeutic citizenship will emerge from, and need to be examined in, specific historical, economic, socio-cultural and political contexts (cf. Nguyen, 2004). We argue that currently, in HICs, therapeutic citizenship is shaped by the patient-centredness and shared decision-making paradigms. Participation in decision-making is a right, and an expectation of the 'good' patient. As Rapley (2008) notes, shared decision-making promotes new collaborative roles, which redistribute rights and responsibilities. Specifically, shared decision-making enables providers to share the burden and transfer some of their responsibility to clients. A provider in Rosengarten et al. (2004, p. 583) notes, talking about side effects 'that you have induced', that 'discussing and letting them [patients] make 
decisions (...) are in part a way of reducing the em burdening guilt if something happens to go wrong'.

The new TasP paradigm may further increase responsibilisation. By widening the treatment remit to those not infected but at risk of HIV, TasP blurs distinctions between the identity categories 'HIV positive' and 'HIV negative', and modifies their associated responsibilities. Members of both categories are now obliged to avoid onward infection, and 'defaulters' may be judged for increasing their sexual partners' infection risks (Keogh \& Dodds, 2015). Thus, we must examine how 'biological citizens may be made increasingly responsible for more than their own health' (Paperini \& Rhodes, 2016, p. 514).

In summary, ART, and the paradigms of patient-centredness and shared decisionmaking, do not merely empower patients; they produce new roles, rights and responsibilities for clients and providers. Clearly, ART and ART adherence support are not merely technical fixes but imbued with social meanings and moral dimensions. These dimensions are ultimately negotiated in the professional-client encounter, and it is to the details of communication in interaction that we now turn.

\subsection{Adherence talk and patient-centredness: The devil is in the interactional detail}

Understanding the aforementioned tensions and complexities requires a detailed examination of adherence talk and provider-client interaction. We need to see how providers actually 'do' trust, patient-centredness and shared decision-making: what conversational strategies do they use to display trust, enact principles of patient-centredness, and achieve shared decisions? How are the tensions and morality at play managed, how are responsibilities and entitlements negotiated and identities produced? Since most studies of ART communication use interviews 
and surveys, they rely on providers' and clients' reports about communication rather than observations of actual interactions. Consequently, broad proxy operationalisations of patientcentredness are common, such as agreement with the statement 'My HIV provider really knows me as a person' (Beach et al., 2006, p. 662). These leave unexplored what communicative behaviours suggest to clients that they are known as a person. Some studies do suggest conversational strategies such as using individualized questions (Tugenberg et al., 2006). However, without detailed analysis of their actual use, studies cannot demonstrate strategies' effects, nor their likely contingency on when in the interaction strategies are used (Barton Laws et al., 2013b). The finding by Callon et al. (2016) that negatively-framed questions (e.g. 'in the past three days would you say that you missed any of the dosages of your medicines?') were 3.64 times more likely to elicit disclosure among non-adherent patients than other types of questions, underscores the importance of conversational details like the exact wording of questions. Finally, most studies focus solely on clients (Sankar, Golin, Simoni, Luborsky, \& Pearson, 2006), rather than on the co-production of meaning and activities between client and provider.

By contrast, joint activities and co-construction are central to our third body of literature: conversation analytic studies of practices used in communication between clients and health professionals (Heritage \& Maynard, 2006). Conversation analysis examines the fine-grained turn-by-turn detail of naturally occurring talk-in-interaction to identify the underlying norms and practices that make those interactions orderly. For example, it examines how speakers take turns or remedy interactional problems; how speakers produce and recipients understand the actions brought about through talk; and how shared understanding is achieved (Sidnell, 2016).

Early CA studies, pre-ART, examined interactions between HIV counsellors and clients, exploring the interactional strategies and devices used to discuss sensitive topics (e.g. 
unsafe sex) in pre- and post-HIV test counselling, or to successfully deliver advice (Kinnell \& Maynard, 1996; Silverman \& Perakyla 1990; Perakyla \& Silverman 1991). Sheon and Lee (2009) explored how counsellors and clients co-construct distrust and uncertainty regarding the partner's disclosure of sero-status, and used their findings to develop training in HIV counselling. Watermeyer and Penn's (2011, p.6) South African study shows how pharmacist assistants may pursue adherence by invoking a client's identity as a community elder with associated responsibilities, or co-construct a client's lack of motivation as basis for nonadherence ('maybe you take this as a game'; 'tell us if not interested'). This may lead to unhelpful and unfair responsibilisation, for instance when external contraints (e.g. financial) limit access to treatment.

Furthermore, there is a growing body of CA research on choice, autonomy and shared decision-making in other healthcare contexts. This work demonstrates firstly that shared decision-making principles are not easily incorporated into all consultations about medicines. Collins et al.'s (2005) comparison of primary care diabetes consultations with secondary care oncology found a spectrum of practitioner approaches, ranging from unilateral to more bilateral. Through detailed sequential analysis, other CA work has shown that, with apparently more bilateral approaches, providers can still limit or expand choice (Pilnick et al., 2004a, b; 2008; Toerien, Shaw \& Reuber, 2013). For example, Pilnick (2004a, 2008) shows how providers' perceptions that they are giving choices regarding antenatal screening for fetal abnormalities are not necessarily matched by clients' experience. Even where practitioners endeavour to adopt patient-centred modes of interaction (for example by encouraging a client to set the agenda for a consultation), there are subtle ways in which choice and decisionmaking can be undermined (e.g. Finlay, Walton \& Antaki, 2008; Pilnick \& Zayts, 2012; Toerien, Shaw \& Reuber, 2013). Conversely, even where more unilateral approaches are used, clients can exert agency in subtle and implicit ways (Gill, 2005). 
CA studies of medical advice-giving illuminate how clients may resist and reject advice (Heritage \& Sefi, 1992; Pilnick, 2003; Stivers, 2007), and how practitioners manage resistance to advice (Emmison, Butler \& Danby 2011; Stivers, 2007). These studies show that direct advice risks being met with resistance. Unsolicited advice-giving can be seen as contrary to client-centredness and empowerment (Emmison, Butler \& Danby, 2011), and as implicating criticism of clients (lack of) actions (Heritage \& Sefi, 1992) or their lack of knowledge (Pilnick, 2003). More subtle forms of advice-giving may thus be more effective. For instance, Butler et al. (2010) show how child helpline counsellors manage the sensitivities of advice-giving by using 'advice-implicative interrogatives', which preserve the client's authority to decide by presenting it in a question format.

Finally, CA studies have shed light on the management of responsibility and morality in clinical encounters, describing for instance how practitioners can hold diabetic clients' accountable for health-related actions (Silverman, 1987), and how clients' can perform moral work by downplaying their agency in treatment failure (Webb, 2009) and framing themselves as 'good patients' (Pilnick \& Coleman, 2003; Webb, 2009).

Taken together then, this third set of literature underscores that perceptions of communication (e.g. as being patient-centred) may differ from actual practices, and that there may be both more and less shared decision-making in ART discussions than surveys and interviews suggest. Furthermore, the CA literature begins to illuminate what concrete communicative strategies may be involved in of patient-centredness and shared decision making (cf. Watermeyer \& Penn, 2011) and why the interactional, joint achievement of these principles is so complex. Finally, CA can elucidate how matters of morality and identity are managed through interaction. 


\section{Discussion}

In summary, psychological and public health studies indicate that provider-client relationships and communication are important for ART adherence and client satisfaction. In particular, trust, patient-centredness and, to a lesser extent, shared decision-making are considered to be beneficial, but not always achieved. Drawing on anthropological and sociological studies, we suggested that this reflects tensions between doing patient-centredness (building trust, sharing decisions, empowering patients), and reaching public health targets, which in turn depends on probing adherence, ensuring desired decisions, and emphasising responsibilities. These tensions are exacerbated by the particular infectious disease and policy context.

The CA literature demonstrated how fine-grained analysis of actual encounters can identify the subtle interactional practices involved in achieving patient-centredness and shared decision-making in practice. CA studies provide details of how, in interaction, through subtle features of what, how and when something is said, speakers can provide choices (or not), offer and resist advice effectively (or not). These techniques, we propose, are ideally suited to an analysis of the enactment of patient-centredness, shared decision-making and trust in ART encounters. CA can provide new insights into the management and resolution of the identified tensions, and illuminate the enactment of rights and responsibilities attached to new forms of therapeutic citizenship, which may well affect patient satisfaction.

Practically, providers have been given little guidance in terms of how to 'do' patientcentredness and shared decision-making in ART adherence support (Watermeyer \& Penn, 2012). General guidelines for practitioners on implementing patient-centredness exist (see e.g. Mathhias et al 2013), but these neglect that interaction and thus patient-centrednesss or shared decision-making are joint activities. CA can help develop strategies which are grounded in this co-production, and so meet the calls for communication training (van den Berg et al., 
2016; Wilson et al., 2010). Whilst the evaluations of CA derived training programmes are still in their infancy, results suggest the potential to bring about changes in communication that are acceptable to both patients and practitioners (Heritage et al., 2007), seemingly because recommendations are rooted in observations of actual talk rather than abstract communication theories or models.

Furthermore, the sociological and anthropological literature illuminated how patientcentredness and shared decision-making may be empowering but can also entail problematic forms of responsibilisation. ART has generated new disease-based, moral identities, expectations, rights and responsibilities, which affect ART adherence and social relationships. Understanding how new forms of therapeutic citizenship are enacted interactionally constitutes a theoretical contribution to $\mathrm{CA}$, and the numerous anthropological and sociological studies of ART, which examine the socio-cultural, economic and political context, but rarely the details of the interactional context. We propose that CA studies engage to a greater extent with theoretical notions developed in the anthropological and sociological literature, and with terms and principles widely used and advocated in policy. Unpacking the interactional specifics of these notions and terms, will illuminate what these mean not only in theoretical terms but also as they are talked into being.

This review has several implications for future studies of ART adherence. They should include a variety of client groups, including those taking ART as prevention. TasP raises new questions, amidst worries that it proceeds without sufficient social science input (Keogh \& Dodds, 2015). Given the increased vulnerability of marginalized patient groups (higher HIV infection rates; less benefit from ART, more loss to follow up (BHIVA, 2016)) and findings that interactions with ethnic minorities exhibited less shared decision-making (Barton Laws et al., 2014), it is important to examine whether and how communication may reflect and contribute to inequalities and marginalization (op cit.). Furthermore, studies should 
study actual interaction, whilst also taking account of the broader social and policy context in which these interactions take place. Incorporating context in CA is not straightforward (de Kok, 2008), but important. Finally, we included a wide range of studies in terms of disciplinary orientation, epistemological and theoretical perspective, and methodology. Recording and synthesizing such a diverse set was challenging and time-consuming, but, importantly, it enabled us to enrich and go beyond the insights generated by any of the three separate sets. Crucially, we applied insights from LMIC studies (e.g. regarding therapeutic citizenship) to HIC settings, which is rarely done: if scholars focus on both settings, the 'transfer' is usually from HIC studies to LMIC studies. More multidisciplinary, multi-context reviews should be conducted in the future, whether regarding ART adherence or other phenomena.

\section{Conclusion}

Supporting ART adherence amongst the growing and increasingly diverse client population remains important yet challenging. Getting HIV care and ART adherence support right is crucial for public health and social justice. Provider-client relationships and communication are undoubtedly important, but understanding whether, when and for whom patientcentredness and shared decision-making are appropriate and feasible requires more detailed interactional studies which also acknowledge the broader social, relational and moral dimensions and effects of ART and ART communication. Further empirical studies which engage with, and integrate, the three literatures reviewed should produce actionable insights which can inform training and policy, leading to better quality professional adherence support. 


\section{References}

Alfonso, V., Geller, J., Bermbach, N., Drummond, A., \& Montaner, J. S. G. (2006).

Becoming a "treatment success": What helps and what hinders patients from achieving and sustaining undetectable viral loads. AIDS Patient Care and STDs, 20(5), 326-334.

Alfonso, V., Toulson, A., Bermbach, N., Erskine, Y., \& Montaner, J. (2009). Psychosocial issues influencing treatment adherence in patients on multidrug rescue therapy: Perspectives from patients and their health care providers. AIDS Patient Care and STDS, 23(2), 119-126.

Altice, F. L., Mostashari, F., \& Friedland, G. H. (2001). Trust and the acceptance of and adherence to antiretroviral therapy. Journal of Acquired Immune Deficiency Syndromes, $28(1), 47-58$.

Ammassari, A., Trotta, M. P., Murri, R., Castelli, F., Narciso, P., Noto, P., ... Antinori, A. (2002). Correlates and predictors of adherence to highly active antiretroviral therapy: Overview of published literature. Journal of Acquired Immune Deficiency Syndromes, 31, S123-S127.

Atkinson, J. S., Schönnesson, L. N., Williams, M. L., \& Timpson, S. C. (2008). Associations among correlates of schedule adherence to antiretroviral therapy (ART): A path analysis of a sample of crack cocaine using sexually active African-Americans with HIV infection. AIDS Care, 20(2), 253-262.

Bader, A., Kremer, H., Erlich-Trungenberger, I., Rojas, R., Lohmann, M., Deobald, O., et al. (2006). An adherence typology: Coping, quality of life, and physical symptoms of people living with HIV/AIDS and their adherence to antiretroviral treatment. Medical. Science Monitor, 12(12), CR493-CR500. 
Ballester, R., Santiago, G., Idoia, R., Campos, A. (2002). Gender differences in adherence to treatment and illness behavior in HIV/AIDS patients: An exploratory study in Spain. International Journal of Psychology \& Psychological Therapy, 2 (2), 219-235.

Barfod, T. S., Hecht, F. M., Rubow, C., \& Gerstoft, J. (2006). Physicians' communication with patients about adherence to HIV medication in San Francisco and Copenhagen: A qualitative study using grounded theory. BMC Health Services Research, 6, 154.

Barton Laws, M., Beach, M. C., Lee, Y., Rogers, W. H., Saha, S., Korthuis, P. T., Wilson, B. (2013a). Provider-patient adherence dialogue in HIV care: Results of a multisite study. AIDS and Behavior, 17(1), 148-159.

Barton Laws, M., Taubin, T., Bezreh, T., Lee, Y., Beach, M. C., \& Wilson, I. B. (2013b). Problems and processes in medical encounters: The cases method of dialogue analysis. Patient Education and Counseling, 91(2), 192-199.

Barton Laws, M., Lee, Y., Rogers, W. H., Beach, M. C., Saha, S., Korthuis, P. T., . . Wilson, I. B. (2014). Provider-patient communication about adherence to anti-retroviral regimens differs by patient race and ethnicity. AIDS and Behavior, 18(7), 1279-1287.

Beach, M. C., Keruly, J., \& Moore, R. D. (2006). Is the quality of the patient-provider relationship associated with better adherence and health outcomes for patients with HIV? Journal of General Internal Medicine, 21(6), 661-665.

BHIVA (2016). BHIVA guidelines for the treatment of HIV-1-positive adults with antiretroviral therapy 2015 (2016 interim update). London: British HIV Association.

Blackstock, O. J., Addison, D. N., Brennan, J. S., \& Alao, O. A. (2012). Trust in primary care providers and antiretroviral adherence in an urban HIV clinic. Journal of Health Care for the Poor and Underserved, 23, 1, 88-98. 
Bruin W.B. de, Hospers, H. J., van Breukelen, G., Kok, G., Koevoets, W. \& Prins, J. M. (2010a). Electronic Monitoring-Based Counseling to Enhance Adherence Among HIVInfected Patients: A Randomized Controlled Trial. Health Psychology, 29, 421-428

Bruin, M., de., Viechtbauer, W., Schaalma, H. P., Kok, G., Abraham, C., \& Hospers, H. J. (2010b). Standard care impact on effects of Highly Active Antiretroviral Therapy Adherence interventions: A meta-analysis of randomized controlled trials. Archives of Internal Medicine, 170, 240-250.

Butler, C.W., Potter, J., Danby, S., Emmison, M., \& Hepburn, A. (2010). Advice implicative interrogatives: Building “client centred” support in a children's helpline. Social Psychology Quarterly 73(3), 265-287.

Callon, W., Saha, S., Korthuis, P.T., Wilson, I.B., Moor, R.D., Cohn, J., \& Beach, M.C. (2016). Which clinician questions elicit accurate disclosure of antiretroviral nonadherence when talking to patients? AIDS Behavior, 20, 1108-1115.

Collins, S., Drew, P., Watt, I. and Entwistle, V. (2005) 'Unilateral' and 'bilateral' practitioner approaches in decision-making about treatment. Social Science \& Medicine, 61, 261127.

Coulter, A. (1997). Partnerships with Patients: The Pros and Cons of Shared Clinical Decision-Making. Journal of Health Services Research Policy, 2(2), 112-121.

Dixon-Woods, M., Cavers, D., Agarwal, S., Annandale, E., Arthur, A., Harvey, J., ...Sutton AJ. (2006). Conducting a critical interpretive synthesis of the literature on access to healthcare by vulnerable groups. BMC Med Res Methodol. 26(6): 35

Emmison, M., Butler, C., \& Danby, S. (2011). Script Proposals: a device for empowering clients in counselling. Discourse Studies, 13(1), 3-26.

Finlay, W.M.L., Walton, C. and Antaki, C. (2008) Promoting choice and control in residential services for people with learning disabilities. Disability and Society, 23(4), 349-60. 
Gilbert, L.M.S.W , Abel, E., Francisco Stewart, N. \& Zilberman, M. (2007). More than drugs. Journal of HIV/AIDS \& Social Services, 6:1-2, 161-179.

Gill, V. (2005). Patient 'demand' for medical interventions: Exerting pressure for an offer in a primary care clinic visit. Research on Language and Social Interaction, 38 (4 ): 451479.

Gough, D., Thomas, \& Oliver, S. (2012) Clarifying differences between review designs and methods. Systematic Review, 1: 28.

Graham, J.L., Shahani, L., Grimes, R.M., Hartman, C. \& Giordano, T.P. (2015). The influence of trust in physicians and trust in the healthcare system on linkage, retention, and adherence to HIV care. AIDS Patient Care and STDs, 29(12), 661-667.

Gupta, R. K., Wainberg, M., Brun-Vezinet, F., Gatell, J. M., Albert, J., Sonnerborg, A., \& Nachega, J. B. (2013). Oral antiretroviral drugs as public health tools for HIV prevention: Global Implications of PrEP for HIV Drug Resistance. Journal of Infectious Diseases 207(S2):S101-6.

Heritage, J. \& Sefi, S. (1992). Dilemmas of advice: Aspects of the delivery and reception of advice in interactions between health visitors and first-time mothers. In P. Drew \& J. Heritage (eds). Talk at work: Interaction in institutional settings (pp. 359- 417). Cambridge: Cambridge University Press.

Heritage, J., \& Maynard, D.W. (2006). Communication in medical care: interaction between primary care physicians and patients. Cambridge: Cambridge University Press.

Heritage, J., Robinson, J.D., Elliott, M.N., Beckett, M. and Wilkes, M. (2007) Reducing patients' unmet concerns in Primary Care: the difference one word can make. Journal of General Internal Medicine, 22(10): 1429-1433 
Ickovics, J. R., \& Meade, C. S. (2002). Adherence to antiretroviral therapy among patients with HIV: A critical link between behavioral and biomedical sciences. Journal of Acquired.Immune.Deficiency.Syndromes, 31 (3), S98-S102.

Kagee, A., Swartz, A., \& Swartz, L. (2014). Theorising beyond the individual: Adherence to antiretroviral therapy in resource-constrained societies. Journal of Health Psychology, 19(1) 103-109.

Kelen, M. \& Cresswell, F. (2017). HIV pre-exposure prophylaxis (PrEP): effective but controversial. Trends Urology \& Men Health, 8: 9-12.

Keogh, P. \& Dodds, C. (2015). Pharmaceutical HIV prevention technologies in the UK: six domains for social science research. AIDS Care, 27:6, 796-803.

Kirwan, P.D., Chau, C., Brown, A.E., Gill, O.N., Delpech, V.C. et al. (2016). HIV in the UK: 2016 report. Public Health England, London.

Kinnell, A. \& Maynard, D. (1996). The delivery of information and advice in pretest counselling session for HIV and AIDS. Journal of contemporary ethnography, 24(4), 405-437.

Kok, B.C. de (2008). The role of context in conversation analysis: Reviving an interest in ethno-methods. Journal of Pragmatics, 40(5), 886-903.

Kok, B.C. de, Laurier, E., Widdicombe, S. (2012). Managing adherence to ART: What lessons can we learn from the analysis of provider-client interactions? Workshop report. Edinburgh: Queen Margaret University. Available at:

http://www.qmu.ac.uk/iihd/docs/2013/WORKSHOPREPORTFINAL3.pdf (accessed 22.6.17)

Kremer, H., Bader, A., O’Cleirigh, C., Bierhoff, H.W., \& Brockmeyer, N.H. (2004). The decision to forgo antiretroviral therapy in people living with HIV - compliance as paternalism or partnership? European Journal of Medical Research, 9(2), 61-70. 
Lewis, M. P., Colbert, A., Erlen, J., \& Meyers, M. (2006). A qualitative study of persons who are $100 \%$ adherent to antiretroviral therapy. AIDS Care, 18(2), 140-148.

Marelich, W. D., \& Murphy, D. A. (2003). Effects of empowerment among HIV-positive women on the patient-provider relationship. AIDS Care - Psychological and SocioMedical Aspects of AIDS/HIV, 15(4), 475-481.

Makoul, G., \& Clayman, M. L. (2006). An integrative model of shared decision making in medical encounters. Patient Education and Counselling, 60(3):301-12.

Matthias, M., Salyers, M. \& Frankel, R. (2013). Re-Thinking Shared Decision-Making: Context Matters. Patient Education and Counseling, 91(2): 176-179.

Mills, E. J., Nachega, J.B., Buchan, I., et al. (2006a). Adherence to antiretroviral therapy in sub-Saharan Africa and North America: a meta-analysis. JAMA (296):679-90

Mills, E. J., Nachega, J. B., Bangsberg, D. R., Singh, S., Rachlis, B., Wu, P., et al. (2006b). Adherence to HAART: A systematic review of developed and developing nation patient-reported barriers and facilitators. PLoS Medicine, 3(11), 2039-2064.

Mol, A. (2008). The logic of care: Health and the problem of patient choice. London: Routledge.

Murri, R., Antinori, A., Ammassari, A., Nappa, S., Orofino, G., Abrescia, N., et al. (2002). Physician estimates of adherence and the patient-physician relationship as a setting to improve adherence to antiretroviral therapy. Journal of Acquired Immune Deficiency Syndromes, 31(3), S158-S162.

Nachega J., Uthman, O., del Rio, C., Mugavero, M., Rees, H., \& Mills, E. J (2014). Addressing the Achilles' Heel in the HIV Care Continuum for the Success of a Testand-Treat Strategy to Achieve an AIDS-Free Generation. Clinical Infectious Diseases, 59(S1): S21-7. 
Nguyen, V. K. (2004). Antiretroviral globalism, biopolitics, and therapeutic citizenship. In: A Ong \& S. Collier (eds.). Global Assemblages: Technology, Politics, and Ethics as Anthropological Problems. London: Blackwell, pp. 124-144.

Nilsson Schonnesson, S. L., Diamond, P.M., Ross M.W., Williams M., Bratt G. (2006). Baseline predictors of three types of antiretroviral therapy (ART) adherence: A 2-year follow-up. AIDS Care, 18(3):246-53.

Novas, C. \& Rose, N. (2000). Genetic risk and the birth of the somatic individual'. Economy and society, 29(4): 485-513.

Oetzel, J., Wilcox, B., Avila, M., Hill, R., Archiopoli, A., \& Ginossar, T. (2015). Patientprovider interaction, patient satisfaction, and health outcomes: testing explanatory models for people living with HIV/AIDS. AIDS Care, 27(8), 972-978.

Okoro, O., \& Odedina, F.T. (2016). Improving medication adherence in African-American women living with HIV/AIDS: Leveraging the provider role and peer involvement. AIDS Care, 28(2), 179-185.

Pach, A.,I, Cerbone, F. G., \& Gerstein, D. R. (2003). A qualitative investigation of antiretroviral therapy among injection drug users. AIDS and Behavior, 7(1), 87-100.

Paperini, S. \& Rhodes, T. (2016). The biopolitics of engagement and the HIV cascade of care: a synthesis of the literature on patient citizenship and antiretroviral therapy. Critical Public Health, 26:5, 501-517.

Pellowski, J.A., Price, D.M., Allen, A.M., Eaton, L.A., \& Kalichman, S.C. (2017). The differences between medical trust and mistrust and their respective influences on medication beliefs and ART adherence among African-Americans living with HIV. Psychology \& Health, 32(9), 1127-1139. 
Perakyla, A. \& Silverman, D. (1991). Reinterpreting Speech-Exchange Systems: Communication Formats in AIDS Counselling. Sociology, 25(4), 627-752.

Pilnick, A. (2003) "Patient counselling" by pharmacists: four approaches to the delivery of counselling sequences and their interactional reception. Social Science and Medicine. 56: 835-49.

Pilnick, A., \& Coleman, T. (2003). “I'll give up smoking when you get me better': patients' resistance to attempts to problematise smoking in general practice (GP) consultations. Social Science \& Medicine, 57, 135-145.

Pilnick, A. (2004a). "It's just one of the best tests that we've got at the moment": informed choice in nuchal translucency screening for fetal abnormality. Discourse and Society, 451-65.

Pilnick, A. (2008). "'It's Something for You Both to Think About'”: Choice and Decision Making in Nuchal Translucency Screening for Downs Syndrome. Sociology of Health and Illness, 30(4), 511-30.

Pilnick, A. \& Dingwall, R. (2011). On the remarkable persistence of asymmetry in doctor/patient interaction: A critical review. Social Science \& Medicine, 72, 1374 1382.

Pilnick, A., \& Zayts, O. (2012). Let's Have It Tested First: Directiveness, Culture and Decision-Making Following Positive Antenatal Screening in Hong Kong. Sociology of Health and Illness, 34(2), 266-82.

Pilnick, A. \& O. Zayts (2015). Advice, authority and autonomy in shared decision making in antenatal screening: The importance of context. Sociology of Health and Illness, 38(3), $343-359$.

Poppa, A., Davidson, O; Deutsch, J., Godfrey, D., Fisher, M., Head, S., Horne, R. and Sherr, L. (2004). British HIV Association (BHIVA)/British Association for Sexual Health and 
HIV (BASHH) guidelines on provision of adherence support to individuals receiving antiretroviral therapy (2003). HIV Medicine (2004), 5 (Suppl. 2), 46-60.

Rapley, T. (2008). Distributed decision making: the anatomy of decisions-in-action. Sociology of Health \& Illness, 30 (3), 429-444.

Roberts, K. J., \& Volberding, P. (1999). Adherence communication: A qualitative analysis of physician-patient dialogue. AIDS, 13(13), 1771-1778.

Roberts, K. J. (2002). Physician-patient relationships, patient satisfaction, and antiretroviral medication adherence among HIV-infected adults attending a public health clinic. AIDS Patient Care STDS, 16(1): 43-50.

Rosengarten, M., Hart, G. Flowers, P. \& Imrie, J. (2004). After the Euphoria: HIV medical technologies from the perspective of their prescribers. Sociology of Health and Illness, 26(5), 575-596.

Saha, S., Jacobs, E.A., Moore, R.D., \& Beach, M.C. (2010). Trust in physicians and racial disparities in HIV care. AIDS Patient Care and STDS, 24(7), 415-420.

Sankar, A., Golin, C., Simoni, J. M., Luborsky, M., \& Pearson, C. (2006). How qualitative methods contribute to understanding combination antiretroviral therapy adherence. Journal of Acquired Immune Deficiency Syndromes, 43, S54-S68.

Schneider, J., Kaplan, S. H., Greenfield, S., Li, W., \& Wilson, I. B. (2004). Better physicianpatient relationships are associated with higher reported adherence to antiretroviral therapy in patients with HIV infection. Journal of General Internal Medicine, 19(11), 1096-1103. 
Scottish Government (2009). HIV Action Plan in Scotland. December 2009 to March 2014. Edinburgh: Scottish Government.

Scottish Parliament (2011). The Patient Rights (Scotland) Act 2011. Edinburgh: Scottish Parliament.

Sheon, N. \& Lee, S. H. (2009). Sero-skeptics: discussions between test counselors and their clients about sexual partner HIV status disclosure. AIDS Care, 221(2):133-9

Sidnell, J. (2016). Conversation Analysis. Oxford Research Encyclopedia of Linguistics. Retrieved from http://linguistics.oxfordre.com/view/10.1093/acrefore/9780199384655.001.0001/ acrefore-9780199384655-e-40.

Siegel,K., Karus, D. \& Schrimshaw, E. W (2000). Racial differences in attitudes toward protease inhibitors among older HIV-infected men. AIDS Care, 12(4), 423-434.

Simoni, J. M., Amico, K. R., Pearson, C. R., and Malow, R. (2008). Strategies for Promoting Adherence to Antiretroviral Therapy: A Review of the Literature. Curr Infect Dis Rep. 10(6): 515-521.

Silverman, D. (1987). Communication and medical practice: Social relations in the clinic. London: Sage.

Silverman, D. \& Perakyla, A. (1990). AIDS counselling: the interactional organisation of talk about 'delicate' issues. Sociology of Health \& Illness, 12(3), 293-318.

Stevenson F.A., Barry C.A., Britten N., Barber N., \& Bradley, C.P. (2000). Doctor-patient communication about drugs: the evidence for shared decision making, Social Science and Medicine, 50(6):829-40.

Stewart, M. (2001). Towards a global definition of patient centred care, British Medical Journal, 322 (7284): 444-445. 
Stivers, T. (2007). Prescribing under Pressure: Parent-Physician Conversations and Antibiotics (Oxford Studies in Sociolinguistics). Oxford: Oxford University Press. Thorburn Bird, S., Bogart, L. M., Delahanty, D. (2004). Health-Related Correlates of Perceived Discrimination in HIV Care. AIDS Patient Care and STDs, 18 (1), 19-26.

Toerien, M., Shaw, R. \& Ruber, M. (2013). Initiating decision-making in neurology consultations: 'recommending' versus 'option-listing' and the implications for medical authority. Sociology of Health \& Illness, 35(6), 873-90.

Tugenberg, T., Ware, N.C., \& Wyatt, M.A. (2006). Paradoxical effects of clinician emphasis on adherence to combination antiretroviral therapy for HIV/AIDS. AIDS Patient Care and STDs, 20(4), 269-274.

UNAIDS (2017). Ending aids: Progress towards the 90-90-90 targets. Geneva: UNAIDS.

Van den Berg, J.J., Neilands, T.B., Johnson, M.O., Chen, B., Saberi, P. (2016). Using path analysis to evaluate the healthcare empowerment model among persons living with HIV for antiretroviral therapy adherence. AIDS Patient Care and STDs, 30(11), 497-505.

Vervoort, S.C.J.M., Borleffs, J.C.C., Hoepelman, A.I.M., \& Grypdonck, M.H.F. (2007). Adherence in antiretroviral therapy: A review of qualitative studies. AIDS, 21(3), 271281.

Ware, N. C., Idoko J., Kaaya S,. Biraro I.A., Wyatt M.A., Agbaji O., Bangsberg, D. R. ... et al. (2009). Explaining Adherence Success in Sub-Saharan Africa: An Ethnographic Study. PLoS Med 6(1): e1000011. https://doi.org/10.1371/journal.pmed.1000011

Watermeyer, J., \& Penn, C. (2012). “Only Two Months Destroys Everything”: A Case Study of Communication About Nonadherence to Antiretroviral Therapy in a South African HIV Pharmacy Context. Health Communication, 27(6), 602-11.

Webb, H. (2009)' 'I've put weight on 'cos I've bin inactive, 'cos I've 'ad me knee done”: moral work in the obesity clinic'. Sociology of Health and Illness 31(6): 854-871. 
WHO (2016). Consolidated guidelines on the use of antiretroviral drugs for treating and preventing HIV infection. Second edition. Geneva: WHO

Wilson, I. B., Laws M.B., Safren S.A., Lee Y., Lu M., Coady W., .. Rogers W., H. (2010). Provider-focused intervention increases adherence-related dialogue but does not improve antiretroviral therapy adherence in persons with HIV. Journal of acquired immune deficiency syndromes, 53(3), 338-47.

Wong, M.D., Cunningham, W.E., Shapiro M.F., Andersen R.M., Cleary P.D., Duan N, ... HCSUS Consortium,. (2004). Disparities in HIV treatment and physician attitudes about delaying protease inhibitors for non- adherent patients. Journal of General Internal Medicine $\_$19(4), 366-374

Yallop, S., Lowth , A., Fitzgerald , M., Reid, J. \& Morelli, A. (2002). The changing world of HIV care: The impact on health professionals. Culture, Health \& Sexuality, 4:4, 431441.

Yin, Z., Brown, A.E., Hughes, G., Nardone, A., Gill O.N, Delpech, V.C. \& contributors (2014). HIV in the United Kingdom 2014 Report. London: Public Health England. 